\title{
Simulation of Multipath Impulse Response for Indoor Wireless Optical Channels
}

\author{
John R. Barry, Joseph M. Kahn, William J. Krause, Edward A. Lee, and David G. Messerschmitt
}

\begin{abstract}
We present a recursive method for evaluating the impulse response of an indoor free-space optical channel with Lambertian reflectors. The method, which accounts for multiple refiections of any order, enables accurate analysis of the effects of multipath dispersion on high-speed indoor optical communication systems. We present a simple algorithm for computer implementation of the technique. We present computer simulation results for both line-of-sight and diffuse transmitter configurations. In both cases, we find that reflections of multiple order are a significant source of intersymbol interference. We also report experimental measurements of optical multipath, which help verify the accuracy of our simulations.
\end{abstract}

\section{INTRODUCTION}

$\mathbf{T}$ HE desire for inexpensive and high-speed data links in wireless local-area network and portable computer applications has prompted the recent interest in indoor optical communication [1]-[12]. A nondirected wireless optical communication system can be categorized as either line-ofsight (LOS) or diffuse. A LOS system is designed under the assumption that the LOS path between transmitter and receiver is unobstructed. We define a diffuse system as one which does not rely upon the LOS path, but instead relies on reflections from a large diffusive reflector such as the ceiling. In both cases, an optical signal in transit from transmitter to receiver undergoes temporal dispersion due to reflections from walls and other reflectors. The intersymbol interference (ISI) that results is a primary impediment to communication at high speeds.

For fixed transmitter and receiver locations, multipath dispersion is completely characterized by an impulse response $h(t)$, defined such that the intensity of the received optical signal is the convolution of $h(t)$ with the intensity of the transmitted optical signal. Mobile transmitters, receivers, and reflectors will result in a time-varying channel, but we will ignore this effect because the channel will vary slowly relative to the bit rate for most indoor applications. In this paper, we present a method for calculating the impulse response of a room with an arbitrarily placed transmitter and receiver. Once calculated, the impulse response can be used to analyze or

Manuscript received February 1992; revised August 1992. This work was supported by the T. J. Watson Research Center, Hawthorne, NY, and by the California State MICRO program. This paper was presented in part at the IEEE Workshop on Wireless Local Area Networks, Worcester, MA, May 1991.

J.R. Barry is with the School of Electrical Engineering, Georgia Institute of Technology, Atlanta, GA 30332-0250.

J. M. Kahn, W. J. Krause, E. A. Lee, and D.G. Messerschmitt are with the Department of EECS, University of California, Berkeley, CA 94720.

IEEE Log Number 9207813. simulate the effects of multipath dispersion on indoor optical communications systems.

Other researchers have modeled indoor reflections of infrared with the purpose of determining the distribution of power throughout a room. Gfeller and Bapst present such an analysis in [2] that accounts for single reflections only; Hash et al. extended the procedure to include double reflections as well [13]. The simulations in these works were meant for a link budget analysis; thus, only the total power reaching the receiver was estimated. In other words, they were concerned only with the time integral of $h(t) .{ }^{1}$ Since power budgets typically have built-in safety margins, the accuracy provided by considering only first- and second-order reflections was sufficient. Hortensius extended the Gfeller and Bapst model to calculate an impulse response, accounting for single reflections only [14].

In contrast to prior work, the method described here can compute the impulse response accounting for any number of reflections. This allows accurate power distribution analysis and, perhaps more importantly, accurate impulse-response analysis. The latter is necessary because signal energy undergoing two or more reflections, although having a reduced amplitude, arrives at the receiver much later than first-order reflections. This temporal spread is critical in high-speed applications, in which case higher-order reflections cannot be ignored.

In the next section, we define the models upon which our procedure is based. In Section III, we describe our recursive algorithm and present a computer implementation. In Section IV, we present simulation results and compare them to experimentally measured results. Finally, to illustrate the impact of the multipath dispersion on system design, we examine the multipath-induced power penalty in Section $\mathrm{V}$.

\section{MODELS}

In this paper, we limit consideration to empty rectangular rooms, although our techniques can be extended to other rooms in a straightforward manner. We next define the models for the source, reflectors, and receiver.

\section{A. Source and Receiver Models}

A wide-beam optical source can be represented by a position vector $\boldsymbol{r}_{S}$, a unit-length orientation vector $\hat{\boldsymbol{n}}_{S}$, a power $P_{S}$,

' For this intensity-in intensity-out channel, the dc value of its frequency response, $H(0)=\int_{-\infty}^{\infty} h(t) d t$, is the fraction of power emitted from a continuous-wave transmitter that is detected by the receiver. 


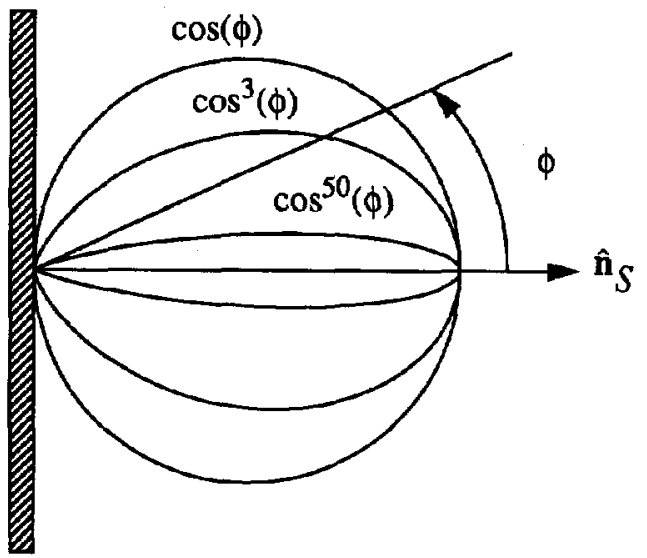

Fig. 1. Normalized shape of the generalized Lambertian radiation pattern.

and a radiation intensity pattern $R(\phi, \theta)$, defined as the optical power per unit solid angle emitted from the source at position $(\phi, \theta)$ with respect to $\hat{\boldsymbol{n}}_{S}$. Following Gfeller [2], we model a source using a generalized Lambertian radiation pattern having uniaxial symmetry (independent of $\theta$ ):

$$
R(\phi)=\frac{n+1}{2 \pi} P_{S} \cos ^{n}(\phi) \quad \text { for } \phi \in[-\pi / 2, \pi / 2] .
$$

Here, $n$ is the mode number of the radiation lobe, which specifies the directionality of the source. This is illustrated in Fig. 1, where sources with higher directionality are seen to have larger mode numbers. The coefficient $(n+1) / 2 \pi$ ensures that integrating $R(\phi)$ over the surface of a hemisphere results in the source power $P_{S}$. A mode of $n=1$ corresponds to a traditional Lambertian source.

To simplify notation, a point source $\mathcal{S}$ that emits a unit impulse of optical intensity at time zero will be denoted by an ordered three-tuple

$$
\mathcal{S}=\left\{\boldsymbol{r}_{S}, \hat{\boldsymbol{n}}_{S}, n\right\}
$$

where $\boldsymbol{r}_{S}$ is its position, $\hat{\boldsymbol{n}}_{S}$ is its orientation, and $n$ in its mode number. Linearity allows us to consider only unit-impulse sources and scale the results for other sources.

Similarly, a receiving element $\mathcal{R}$ with position $r_{R}$, orientation $\hat{\boldsymbol{n}}_{R}$, area $A_{R}$, and field of view $F O V$ will be denoted by an ordered four-tuple

$$
\mathcal{R}=\left\{r_{R}, \hat{n}_{R}, A_{R}, F O V\right\} .
$$

The scalar angle $F O V$ is defined such that a receiver only detects light whose angle of incidence (with respect to the detector normal $\hat{\boldsymbol{n}}_{R}$ ) is less than FOV. A limited field of view may be an inadvertent effect of detector packaging, or it may be used intentionally to reduce unwanted reflections or noise.

\section{B. Reflector Model}

Although true reflections contain both specular and diffusive components [15], we make the simplifying assumption that all reflectors are purely diffusive ideal Lambertian. Experimental measurements have shown that many typical materials such as plaster walls, acoustic-tiled walls, carpets, and unvarnished wood are well-approximated as Lambertian [2], [13], [18].

The radiation intensity pattern $R(\phi)$ emitted by a differential element of an ideal diffuse reflector is independent of the angle of the incident light. This fact is key to our results because it allows us to decompose a reflection into two sequential steps: to model the reflection from a differential reflecting element with area $d A$ and reflectivity $\rho$, first consider the element as a receiver with area $d A$ and calculate the power $d P$ it receives. Second, model the differential reflector as a source with total power $P=\rho d P$ and an ideal Lambertian radiation intensity pattem, as given by (1) with $n=1$.

\section{Line-of-Sight Impulse Response}

Consider a source $\mathcal{S}$ and receiver $\mathcal{R}$, as specified by (2) and (3), in an environment with no reflectors (see Fig. 2). If the distance $R$ between a transmitter and receiver is large relative to the detector size, so that $R^{2} \gg A_{R}$, then the received irradiance is approximately constant over the surface of the detector. Furthermore, all of the signal energy will arrive at the receiver at approximately the same time. Thus, using the models described previously, the impulse response for this simple system is approximately a scaled and delayed Dirac delta function

$h^{(0)}(t ; \mathcal{S}, \mathcal{R}) \approx \frac{n+1}{2 \pi} \cos ^{n}(\phi) d \Omega \operatorname{rect}(\theta / F O V) \delta(t-R / c)$

where $d \Omega$ is the solid angle subtended by the receiver's differential area (assuming $A_{R} \ll R^{2}$ )

$$
d \Omega \approx \cos (\theta) A_{R} / R^{2}
$$

$R$ is the distance between the source and receiver

$$
R=\left\|r_{S}-r_{R}\right\|
$$

$\theta$ is the angle between $\hat{\boldsymbol{n}}_{R}$ and $\left(\boldsymbol{r}_{S}-\boldsymbol{r}_{R}\right)$

$$
\cos (\theta)=\hat{\boldsymbol{n}}_{R} \cdot\left(\boldsymbol{r}_{S}-\boldsymbol{r}_{R}\right) / R
$$

$\phi$ is the angle between $\hat{n}_{S}$ and $\left(r_{R}-r_{S}\right)$

$$
\cos (\phi)=\hat{n}_{S} \cdot\left(\boldsymbol{r}_{R}-\boldsymbol{r}_{S}\right) / R
$$

the rectangular function is defined by:

$$
\operatorname{rect}(x)= \begin{cases}1 & \text { for }|x| \leq 1 \\ 0 & \text { for }|x|>1\end{cases}
$$

and $c$ is the speed of light. The approximation of (4) approaches equality as the ratio $A_{R} / R^{2}$ approaches zero.

\section{Multiple-Bounce IMPULSE ResPonse}

We now describe our algorithm for calculating a multiplebounce impulse response, after which we discuss a computer implementation. 


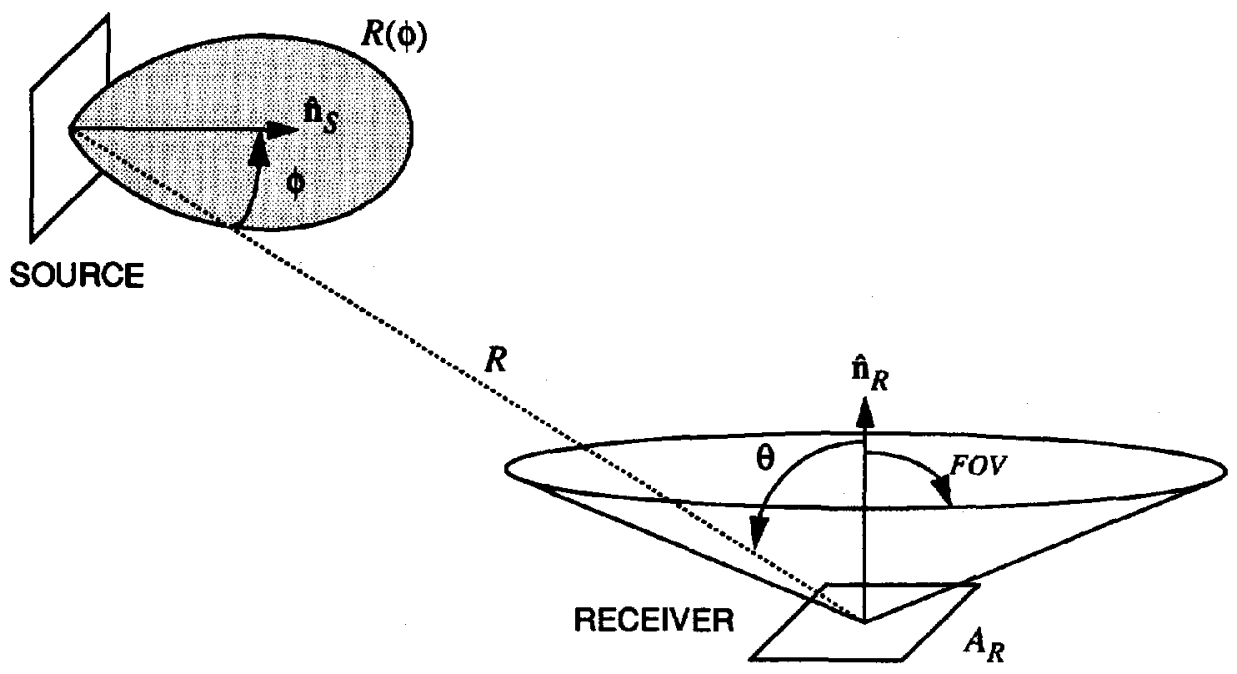

Fig. 2. Geometry of source and detector, without reflectors.

\section{A. Algorithm}

Given a particular source $\mathcal{S}$ and receiver $\mathcal{R}$ in a room with reflectors, light from the source can reach the receiver after any number of reflections. Therefore, the impulse response can be written as an infinite sum:

$$
h(t ; \mathcal{S}, \mathcal{R})=\sum_{k=0}^{\infty} h^{(k)}(t ; \mathcal{S}, \mathcal{R})
$$

where $h^{(k)}(t)$ is the response of the light undergoing exactly $k$ reflections. The line-of-sight response $h^{(0)}(t)$ is given by (4), while higher-order terms $(k>0)$ can be calculated recursively:

$$
\begin{aligned}
h^{(k)}(t ; \mathcal{S}, \mathcal{R})= & \int_{S} h^{(0)}\left(t ; \mathcal{S},\left\{\boldsymbol{r}, \hat{\boldsymbol{n}}, \pi / 2, d r^{2}\right\}\right) \\
& \otimes h^{(k-1)}(t ;\{\boldsymbol{r}, \hat{\boldsymbol{n}}, 1\}, \mathcal{R})
\end{aligned}
$$

where the symbol $\otimes$ denotes convolution. More explicitly, substituting (4) and performing the convolution results in

$$
\begin{aligned}
h^{(k)}(t ; \mathcal{S}, \mathcal{R}) & =\frac{n+1}{2 \pi} \int_{S} \frac{\rho_{\boldsymbol{r}} \cos ^{n}(\phi) \cos (\theta)}{R^{2}} \\
& \cdot \operatorname{rect}(2 \theta / \pi) h^{(k-1)}(t-R / c ;\{\boldsymbol{r}, \hat{\boldsymbol{n}}, 1\}, \mathcal{R}) d \boldsymbol{r}^{2}
\end{aligned}
$$

The integrations in (11) and (12) are performed with respect to $r$ on the surface $S$ of all reflectors. Here, $\hat{n}$ is the normal to the surface $S$ at position $r, \rho_{r}$ is the reflectivity at position $\boldsymbol{r}, R=\left\|\boldsymbol{r}-\boldsymbol{r}_{S}\right\|, \cos (\phi)=\hat{\boldsymbol{n}}_{S} \cdot\left(\boldsymbol{r}-\boldsymbol{r}_{S}\right) / R$, and $\cos (\theta)=$ $\hat{\boldsymbol{n}} \cdot\left(\boldsymbol{r}_{S}-\boldsymbol{r}\right) / \boldsymbol{R}$.

Equation (11) is our main theoretical result. Intuitively, it says that the $k$-bounce impulse response from a single pointsource $\mathcal{S}$ can be found by first finding the distribution and timing of the power from $\mathcal{S}$ onto the reflecting walls. Then, using the walls as a distributed light source, computing the $(k-1)$-bounce impulse response.

\section{B. Implementation}

The integral in (11) can be calculated numerically by breaking the reflecting surfaces into numerous small reflecting elements, each with area $\Delta A$. Thus, $h^{(k)}(t)$ can be approximated by:

$$
\begin{aligned}
h^{(k)}(t ; \mathcal{S}, \mathcal{R}) \approx & \sum_{i=1}^{N} h^{(0)}\left(t ; \mathcal{S}, \mathcal{E}_{i}\right) \otimes h^{(k-1)}\left(t ; \mathcal{E}_{i}, \mathcal{R}\right) \\
= & \frac{n+1}{2 \pi} \sum_{i=1}^{N} \frac{\rho_{i} \cos ^{n}(\phi) \cos (\theta)}{R^{2}} \\
& \cdot \operatorname{rect}(2 \theta / \pi) h^{(k-1)}(t-R / c ;\{r, \hat{n}, 1\}, \mathcal{R}) \Delta A
\end{aligned}
$$

where $\mathcal{E}_{i}$ signifies the $i$ th element and $N$ is the total number of elements. Note how $\mathcal{E}_{i}$ plays the role of both an elemental receiver and an elemental source. This spatial discretization will cause temporal discretization as well, turning the normally piecewise-continuous function of time $h^{k}(t)$ into a finite sum of scaled delta functions; temporal smoothing can be achieved by subdividing time into bins of width $\Delta t$ and summing the total power received in each bin. ${ }^{2}$ The resulting histogram closely approximates the actual $h^{(k)}(t)$, achieving equality as $\Delta A$ and $\Delta t$ approach zero.

Direct implementation of (13) is not efficient for reflection orders $k$ greater than 1 , because identical computations would then be performed multiple times. To see this, consider a room with its reflectors subdivided into a total of $N$ elements. Then, in calculating (13), a total of $N^{k}$ elementary computations are performed, where one elementary computation is defined as the calculation of differential power and delay from a point source to a differential receiver, as in (4). Thus, an elementary computation consists of the collection of multiplications and vector

${ }^{2}$ Empirical evidence suggests that a good choice for the bin width is $\Delta t=\sqrt{\Delta A} / c$, which is roughly the time it takes for light to travel between neighboring elements. 
dot-products described in (5)-(9). However, there are only $(N+1)^{2}$ unique elementary computations, corresponding to the line-of-sight impulse response from any element (including source) to any element (including receiver). Therefore, a more efficient approach would be to construct two lookup tables, each consisting of $(N+1)^{2}$ entries. The first, call it $d P(i, j)$, should contain the differential power between element $i$ and element $j$. The second, call it $\tan (i, j)$, should contain the delay between element $i$ and element $j$. With these two tables, a numerical procedure for calculating the $k$-bounce impulse response is easily implemented, as illustrated by the following pseudocode.

$$
\begin{aligned}
& \text { function } h(t ; i, j, k) \\
& \text { begin } \\
& \text { if }(k=0) \\
& \quad \text { return } d P(i, j) \times \text { delta }(t-\operatorname{tau}(i, j)) \\
& \text { else } \\
& \quad \text { return sum from } e=1 \text { to } N \\
& \quad \text { rho }(e) \times d P(i, e) \times h(t-\operatorname{tau}(i, e) ; e, j, k-1)
\end{aligned}
$$$$
\text { end }
$$

Here, $h(t ; i, j, k)$ is a function that returns the $k$-bounce impulse response $h^{(k)}(t)$ with element $i$ as the source and element $j$ as the receiver. The reflectivity $\rho_{e}$ of the $e$ th reflecting element is given by rho(e). This algorithm is applicable to rooms of arbitrary shape, although in the next section we present results for empty rectangular rooms only.

The table lookup approach requires roughly $8 N^{2}$ bytes of memory (assuming a floating-point precision of 4 bytes per entry). Thus, a modest number of reflectors can lead to prohibitive memory requirements. For example, a $10 \mathrm{~m} \times$ $10 \mathrm{~m} \times 5 \mathrm{~m}$ room with a spatial resolution of $\Delta A=100 \mathrm{~cm}^{2}$ has $N=4 \times 10^{4}$ elements and would thus require 12.8 Gbytes of storage. To meet a more realistic storage limit of 32 Mbytes, the number of elements must satisfy $N<2 \times 10^{3}$ or, equivalently, $\Delta A>0.2 \mathrm{~m}^{2}$.

When the number of elements exceeds the limit imposed by memory restrictions, the direct approach must be used. The same algorithm outlined in pseudocode is applicable to the direct approach, except that each occurrence of $d P(i, j)$ and $\operatorname{tau}(i, j)$ must be calculated anew.

The time required to compute $h^{(k)}(t)$ is roughly proportional to the number of bottom-level function calls, which from inspection of the pseudocode is $N^{k}$. The run time is thus exponential in $k$, which severely limits the number of refiections that can be computed in a reasonable amount of time. Using the table lookup approach in the $\mathrm{C}$ programming language on a Sun Sparcstation 2, we derived an empirical runtime estimate of $N^{k} \times 4 \mu \mathrm{s}$. Thus, for example, to compute the $k=3$ bounce impulse response with $N=2776$ elements (these numbers are extracted from the results of the next section-see the last three columns of Table I), the run time is roughly 24 hours, whereas to compute the $k=4$ bounce impulse response with the same number of elements would require about $N$ days or 7.5 years. Reducing $N$ would shorten the run time at the expense of reduced accuracy. We resist this temptation and present results for reflections up to third-order only.

\section{RESULTS}

In the next two subsections, we present impulse responses from both simulation and experimental measurement. These impulse responses $h(t)$ are defined as the received optical intensity when the transmitted optical intensity is a unitarea Dirac delta function. Therefore, the dc gain $H(0)=$ $\int_{-\infty}^{\infty} h(t) d t$ is related to the average received power $P_{r}$ by $P_{r}=P_{T} H(0)$, where $P_{T}$ is the average transmitted power. We will find it convenient to compare results under the assumption that the transmitted power is $1 \mathrm{~W}$, and so we often associate the integral of $h(t)$ with the average received power due to a $1 W$ transmitter.

\section{A. Simulation Results}

A computer program was written that implements the algorithm described in the previous section. The user can specify the various parameters listed in Table I. We equate north with $\hat{\boldsymbol{x}}$ and west with $\hat{y}$. The elevation angles are defined with respect to the horizontal plane, so that a source pointing straight down has an elevation of $-90^{\circ}$, and a receiver pointing straight up has an elevation of $90^{\circ}$. The azimuth angle at position $r$ is defined as the angle between $\hat{x}$ and the projection of $r$ onto the $x-y$ plane, with a sign defined so that $\hat{y}$ has an azimuth of $90^{\circ}$. The final set of parameters in the table control the resolution of the simulation. Here, $\Delta t$ is the bin width of the power histogram that approximates the impulse response, and bounces is the number of reflections that are considered. The spatial resolution of the simulation is specified by the number of partitions per dimension. The total number of differential reflecting elements is then given by

$$
N=2\left(N_{x} N_{y}+N_{x} N_{z}+N_{y} N_{z}\right)
$$

In Fig. 3, we show the simulated impulse responses $h^{(k)}(t), k \in\{0,1,2,3\}$, for the configuration $A$ given in the first column of Table I. (We consider the other columns in the next section.) We were able to use higher spatial resolutions for the lower-order reflections, because their run times are short. The number of partitions per dimension for each bounce are indicated in the last three rows of Table I. The time origin is defined by the arrival of the line-of-sight impulse. Each of the responses is labeled by the total power it would carry if the source emitted $1 \mathrm{~W}$ in continuous-wave mode. Thus, $h^{(0)}(t)$ is a delta function, scaled by $1.23 \times 10^{-6}$. The numbers in parenthesis specify the percentage of power due to that pulse. The first-order response is seen to have four peaks, corresponding to the four walls of the room; assuming a 1-W source, the total power from once-reflected light is $0.505 \mu \mathrm{W}$. Total power is seen to decrease for each of the higher-order impulse responses; however, they tend to add to a significant amount, as shown in the sum impulse response at the bottom of the figure. Furthermore, this power arrives much later than that from lower-order reflections.

The net result is that higher-order reflections are significant. This may be easier to see in the frequency domain. Using the results of Fig. 3, we can estimate the frequency response of 
TABLE I

PARAMETERS FOR SIMULATION AND EXPERIMENT

\begin{tabular}{|c|c|c|c|c|c|c|c|c|c|c|c|c|c|}
\hline \multicolumn{2}{|c|}{ parameter } & \multicolumn{3}{|c|}{ Configuration $\mathbf{A}$} & \multicolumn{3}{|c|}{ Configuration B } & \multicolumn{3}{|c|}{ Configuration $\mathrm{C}$} & \multicolumn{3}{|c|}{ Configuration D } \\
\hline \multirow[t]{9}{*}{ room: } & length $(x)$ & \multicolumn{3}{|c|}{$5 \mathrm{~m}$} & \multicolumn{3}{|c|}{$7.5 \mathrm{~m}$} & \multicolumn{3}{|c|}{$7.5 \mathrm{~m}$} & \multicolumn{3}{|c|}{$7.5 \mathrm{~m}$} \\
\hline & width $(y)$ & \multicolumn{3}{|c|}{$5 \mathrm{~m}$} & \multicolumn{3}{|c|}{$5.5 \mathrm{~m}$} & \multicolumn{3}{|c|}{$5.5 \mathrm{~m}$} & \multicolumn{3}{|c|}{$5.5 \mathrm{~m}$} \\
\hline & height ( $(z)$ & \multicolumn{3}{|c|}{$3 \mathrm{~m}$} & \multicolumn{3}{|c|}{$3.5 \mathrm{~m}$} & \multicolumn{3}{|c|}{$3.5 \mathrm{~m}$} & \multicolumn{3}{|c|}{$3.5 \mathrm{~m}$} \\
\hline & $\rho_{\text {NORTH }}$ & \multicolumn{3}{|c|}{0.8} & \multicolumn{3}{|c|}{0.30} & \multicolumn{3}{|c|}{0.58} & \multicolumn{3}{|c|}{0.58} \\
\hline & PSOUTH & \multicolumn{3}{|c|}{0.8} & \multicolumn{3}{|c|}{0.56} & \multicolumn{3}{|c|}{0.56} & \multicolumn{3}{|c|}{0.56} \\
\hline & $\rho_{E A S T}$ & \multicolumn{3}{|c|}{0.8} & \multicolumn{3}{|c|}{0.30} & & 0.30 & & & 0.30 & \\
\hline & $\rho_{W E S T}$ & & 0.8 & & & 0.12 & & & 0.12 & & & 0.12 & \\
\hline & PCEILING & & 0.8 & & & 0.69 & & & 0.69 & & & 0.69 & \\
\hline & $\rho_{F L O O R}$ & & 0.3 & & & 0.09 & & & 0.09 & & & 0.09 & \\
\hline source: & mode & & 1 & & & 1 & & & 1 & & & 1 & \\
\hline & $\mathbf{x}$ & & 2.5 & & & 2.0 & & & 5.0 & & & 3.75 & \\
\hline & $y$ & & 2.5 & & & 4.0 & & & 1.0 & & & 2.75 & \\
\hline & $\mathbf{z}$ & & 3 & & & 3.3 & & & 3.3 & & & 1.0 & \\
\hline & elevation & & $-90^{\circ}$ & & & $-90^{\circ}$ & & & $-70^{\circ}$ & & & $+90^{\circ}$ & \\
\hline & azimuth & & $0^{\circ}$ & & & $0^{*}$ & & & $10^{\circ}$ & & & $0^{\circ}$ & \\
\hline receiver: & area & & $1 \mathrm{~cm}^{2}$ & & & $\overline{1 \mathrm{~cm}^{2}}$ & & & $1 \mathrm{~cm}$ & & & $1 \mathrm{~cm}^{2}$ & \\
\hline & FOV & & $85^{\circ}$ & & & $70^{\circ}$ & & & $70^{\circ}$ & & & $70^{\circ}$ & \\
\hline & $\mathrm{x}$ & & $0.5 \mathrm{~m}$ & & & $6.6 \mathrm{~m}$ & & & $2.0 \mathrm{~m}$ & & & $6.0 \mathrm{~m}$ & \\
\hline & $y$ & & $1.0 \mathrm{~m}$ & & & $2.8 \mathrm{~m}$ & & & $4.0 \mathrm{~m}$ & & & $0.8 \mathrm{~m}$ & \\
\hline & $\mathbf{z}$ & & $0 \mathrm{~m}$ & & & $0.8 \mathrm{~m}$ & & & $0.8 \mathrm{n}$ & & & $0.8 \mathrm{~m}$ & \\
\hline & elevation & & $90^{\circ}$ & & & $90^{\circ}$ & & & $90^{\circ}$ & & & $90^{\circ}$ & \\
\hline & azimuth & & $0^{\circ}$ & & & $0^{\circ}$ & & & $0^{*}$ & & & $0^{*}$ & \\
\hline resolution: & & & $0.2 \mathrm{~ns}$ & & & $0.2 \mathrm{n}$ & & & $0.2 \mathrm{n}$ & & & $0.2 \mathrm{n}$ & \\
\hline & bounces & 1 & 2 & 3 & 1 & 2 & 3 & 1 & 2 & 3 & 1 & 2 & 3 \\
\hline & $N_{x}$ & 500 & 100 & 25 & 750 & 150 & 30 & 750 & 150 & 30 & 750 & 150 & 30 \\
\hline & $N_{y}$ & 500 & 100 & 25 & 550 & 110 & 22 & 550 & 110 & 22 & 550 & 110 & 22 \\
\hline & $N_{z}$ & 300 & 60 & 15 & 350 & 70 & 14 & 350 & 70 & 14 & 350 & 70 & 14 \\
\hline
\end{tabular}

the channel

$$
\begin{aligned}
H(\omega) & =\int_{\infty}^{\infty} h(t) e^{-j \omega t} d t \approx \sum_{n=-\infty}^{\infty} h(n \Delta t) e^{j \omega n \Delta t} \\
& =\Delta t H\left(e^{j \omega \Delta t}\right)
\end{aligned}
$$

where $H\left(e^{j \omega \Delta t}\right)$ is the discrete-time Fourier transform of the discrete-time signal $h(n \Delta t)$. In Fig. 4 , we plot $\left|\Delta t H\left(e^{j \omega \Delta t}\right)\right|$ versus $\omega$ for impulse responses accounting for up to zero through three reflections. In other words, we approximate $h(t)$ by replacing the upper limit in the summation of (10) by $K \in\{0,1,2,3\}$. The curves in Fig. 4(a) show how the higher-order reflections increase the dc component of the frequency response while decreasing its component at other frequencies. The $\mathrm{dc}$ gain of $-112.3 \mathrm{~dB}=20 \log _{10}\left(2.4 \times 10^{-6}\right)$ for $K=3$ implies that the receiver detects $2.4 \mu \mathrm{W}$ for a 1
W continuous-wave transmitter. From Fig. 4(a), we also see that the higher-order reflections have significant impact only at low frequencies; the high-frequency magnitude response is characterized by the first-order reflection only.

To highlight the effects of higher-order bounces on the -3 $\mathrm{dB}$ bandwidth, we normalize the magnitude responses to have unity de gain in Fig. 4(b). This figure illustrates the need for considering higher-order reflections. Each additional reflection tends to lengthen the duration of the impulse response, which decreases the bandwidth of the channel. The $K=1$ channel has a $-3 \mathrm{~dB}$ bandwidth $30 \mathrm{MHz}$, while the $K=3$ channel has a bandwidth of only $9 \mathrm{MHz}$.

In Fig. 5, we show the phase response and group-delay response of the channel. The group delay is defined as $-(\partial / \partial \omega) \angle H(\omega)$, so that a linear-phase channel has constant delay. The line-of-sight impulse response, being a delta function, has zero phase and thus zero delay. As the number 

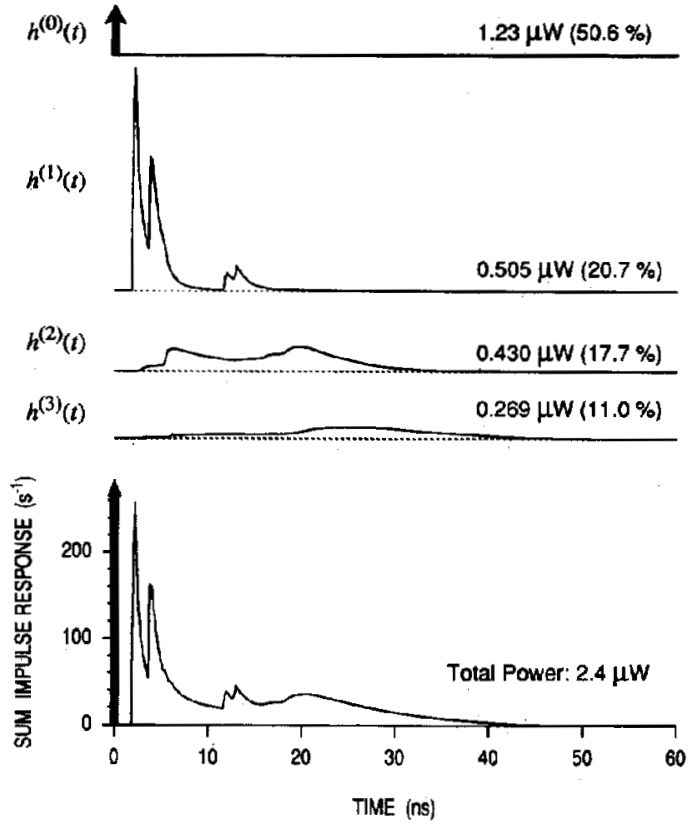

Fig. 3. Impulse responses for light undergoing $k \in\{0,1,2,3\}$ reflections and their $\operatorname{sum}$ (for configuration $\mathrm{A}$, assuming a source power of $1 \mathrm{~W}$ ).

of reflection increases, the phase response becomes less linear, and the delay response exhibits more variability.

\section{B. Experimental Results and Comparison with Simulation}

In this subsection, we present simulation results and compare them with experimental results for the room described in the last three columns of Table 1 . This is an empty conference room with a wide variety of reflecting materials, including textured acoustic-tiled walls (east wall, north wall for configuration B only), rugs (floor, west wall), wood (doors on north and south walls), and painted plaster (remainder of south wall). For configurations $C$ and $D$, we covered the north wall with a white projection screen. The reflectivities of each of these surfaces were measured experimentally as follows: light from a laser was directed towards the material under test at normal incidence, and the reflected power was measured, also at normal incidence. The reflectivity was then chosen so that an ideal Lambertian reflector with identical reflectivity would yield the same reflected power. The results of the reflectivity measurements are shown in the table.

The transmitter in our experimental setup was a $832 \mathrm{~nm}$ laser diode with peak power of $100 \mathrm{~mW}$. The laser was enclosed in a metal box and illuminated a $3 \times 10 \mathrm{~cm}$ area on a translucent plexiglass window, which emitted a broad optical beam with an approximately Lambertian radiation pattern. The receiver front end consisted of a $0.25 \mathrm{~cm}^{2} \mathrm{Si}$ avalanche photodiode and a transimpedance amplifier. We measured the frequency response of the channel using a $300 \mathrm{kHz}-3 \mathrm{GHz}$ vector network analyzer. Td lfriprove noise immunity, we turned off the lights during medsurements and used a small IF bandwidth for averaging. To isoldte the desired frequency response of the optical channel from that of the measurement
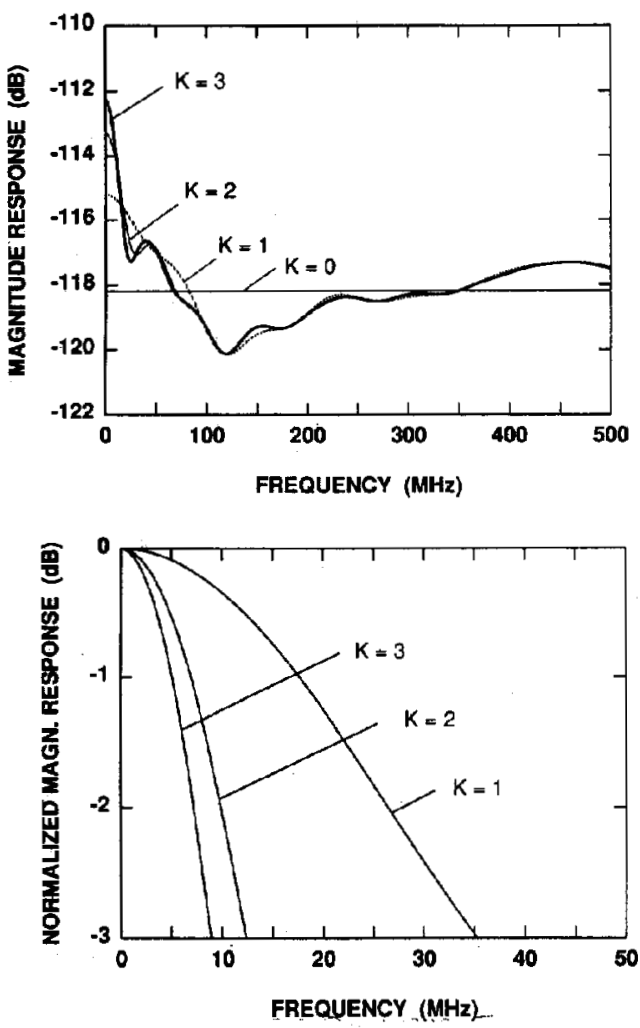

Fig. 4. Effects of high-order reflections on frequency response (configuration A).

system, each measured frequency response was divided by the frequency response of the measurement system. To prevent multipath from corrupting the measurement of the frequency response of the measurement system, a thin $1 \mathrm{~m}$ tube was held between the transmitter and receiver during calibration.

1) Configuration B: Consider the scenario in the second column of Table I, entitled configuration B. Like configuration $A$, this is also a LOS system, but here the room is somewhat larger and its walls have lower reflectivities. The transmitter is mounted near the ceiling in the southwest corner, pointing straight down, with a mode of $n=1$ (ideal Lambertian). The receiver is at the north end of the room pointing straight up. In Fig. 6, we show simulation results using the time and spatial resolution specified in Table I. In Fig. 6(a), we show separately each of the $k$-bounce pulses for $k \in\{0,1,2,3\}$. The LOS impulse arrives at time zero and carries $78 \%$ percent of the total power, which is $307 \mathrm{nW}$ for a $1 \mathrm{~W}$ source. It is interesting to note that the second-order bounce carries about $3.4 \mathrm{~dB}$ more power than the first-order bounce. This is likely due to the fact that it takes two bounces for light to reflect from the highly reflective ceiling, which fills the receiver's field of view. The sum of the pulses in Fig. 6(a) yields the total impulse response of Fig. 6(b). The total power of $307 \mathrm{nW}$ is about $9 \mathrm{~dB}$ less than the received power for configuration $A$, the primary reason being that, here, the distance between transmitter and receiver is larger. This illustrates the strict limits on coverage area imposed by path loss.

Experimental measurements were also performed using the 

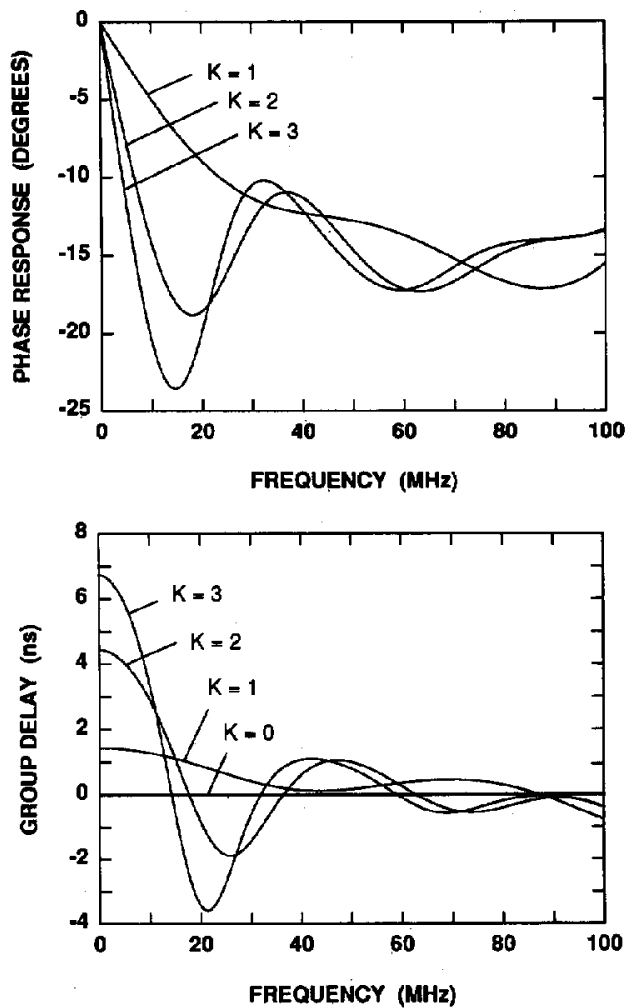

Fig. 5. Effects of high-order reflections on phase (a) and delay response (b) for configuration $A$.

configuration B parameters of Table I. In Fig. 6(c), we illustrate the magnitude response of the experimental channel (solid curve) and of the simulated channel (dashed curve). The experimental and simulation results are seen to agree qualitatively; both exhibit high dc gain and oscillatory highfrequency components. This structure is, in fact, common for all multipath channels consisting of a Dirac delta function plus a lowpass impulse response "tail." The experimental and simulation results do not coincide precisely, however. Two reasons stand out as likely candidates. First, we considered only reflections up to third order in our simulations; as illustrated in Fig. 4(a), higher-order reflections tend to emphasize the low-frequency components and deemphasize the "notch" frequencies, which would make the simulation result look more like the experimental one. Second, our simulations were based on idealized models of the reflectors, so any discrepancies between simulation and experiment may be attributable to nonideal or specular reflectors. Other possible reasons for discrepancy include angle-dependent reflections at the air-detector interface and a nonideal transmitter radiation pattern.

The $-3 \mathrm{~dB}$ bandwidth of the experimental channel is 14 $\mathrm{MHz}$, which is close to the $19.5 \mathrm{MHz}$ predicted by simulation. The bandwidth results for configurations $A$ through $D$ are summarized in Table II.

A time-domain representation of the experimentally measured frequency response can be obtained by performing an inverse Fourier transform. To minimize the effects of the noise, which from Fig. 6(a) are seen to dominate at high frequencies, we used a $250 \mathrm{MHz}$ Hamming window before performing the inverse transform. The solid curve in Fig. 6(d) shows the resulting time-domain signal. The dashed curve was obtained from the frequency response of the simulated impulse response in the same manner, i.e., by windowing and inverse transforming. This process removes much of the structure of the original impulse response of Fig. 6(b), but facilitates comparison between simulation and experiment. The timedomain signals are seen to agree reasonably well. The full width at half-maximum of the main pulse is about $4 \mathrm{~ns}$, which is the minimum resolution offered by the $250 \mathrm{MHz}$ window. The experimental impulse response exhibits a larger multipath tail than the simulated impulse response.

It is no accident that the experimental curve achieves precisely the same maximum value as the simulation curve in Fig. 6(d). In fact, this was how we calibrated the experimental data. The underlying assumption is that our simulation model for LOS path loss matches that of the experiment. This is a reasonable assumption, given that the LOS path loss is governed by the geometry of the configuration only, which, unlike nonideal reflectors, is easily specified.

2) Configuration $C$ : Configuration $C$, like configurations $A$ and $B$, is the LOS system with the transmitter mounted on the ceiling. Here, however, the acoustic tiles on the north wall were covered by a highly reflective white projection screen, and the transmitter was pointed not straight down but at an elevation of $-70^{\circ}$ and an azimuth of $10^{\circ}$.

In Fig. 7(a), we show each of the $k$-bounce pulses for $k \in$ $\{0,1,2,3\}$ as predicted by simulation. Note again that, as in configuration $\mathbf{B}$, the second-order bounce carries more power than the first-order bounce, this time by about $5.8 \mathrm{~dB}$. The pulses in Fig. 7(a) combine to yield the total impulse response of Fig. 7(b). The total received power for a $1 \mathrm{~W}$ source is $283.7 \mathrm{nW}$, about $0.3 \mathrm{~dB}$ less than that for configuration $\mathrm{B}$ (see Table II).

The experimentally measured frequency response for configuration B is compared to the simulated response in Fig. 7(c). The two curves have the same general shape, but again the simulations seem to underestimate the low-frequency components of the channel, perhaps because only reflections up to third order were considered. The experimental $-3 \mathrm{~dB}$ bandwidth of $12 \mathrm{MHz}$ is in good agreement with the simulation bandwidth of $13.4 \mathrm{MHz}$.

The time-domain comparison between simulation and experiment is presented in Fig. 7(d), using a procedure identical to that of Fig. 6(d). As before, the experimental impulse response exhibits a larger multipath contribution than the simulated impulse response.

3) Configuration D: In contrast to the configurations considered so far, configuration $D$ of the fourth column of Table 1 represents a diffuse system, with the transmitter in the center of the room near the floor and aimed towards the ceiling. This is similar to the original configuration first proposed by Gfeller over a decade ago [1]. The primary advantage of the diffuse approach is its inherent robustness to shadowing. By illuminating the ceiling with a broad optical beam, the 


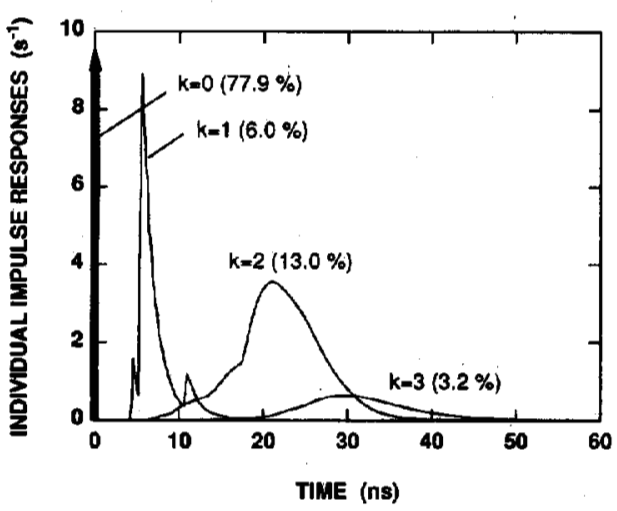

(a)

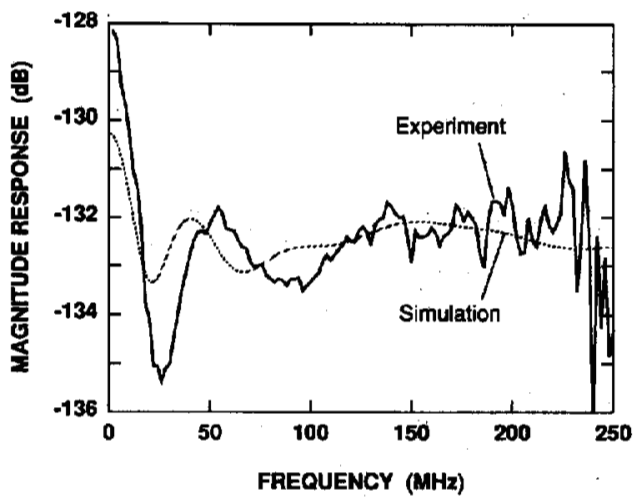

(c)

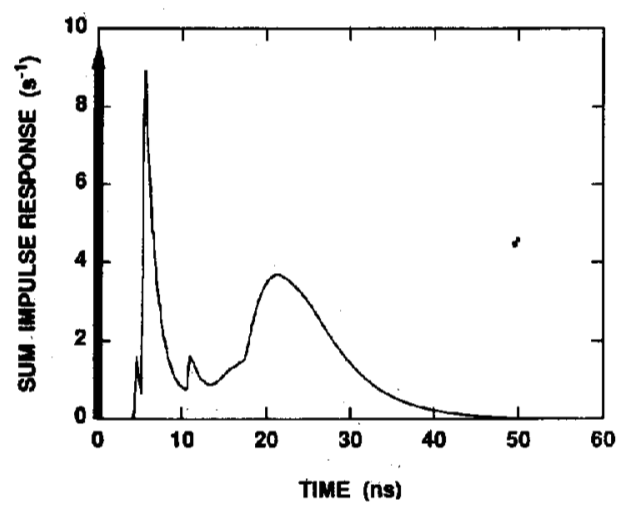

(b)

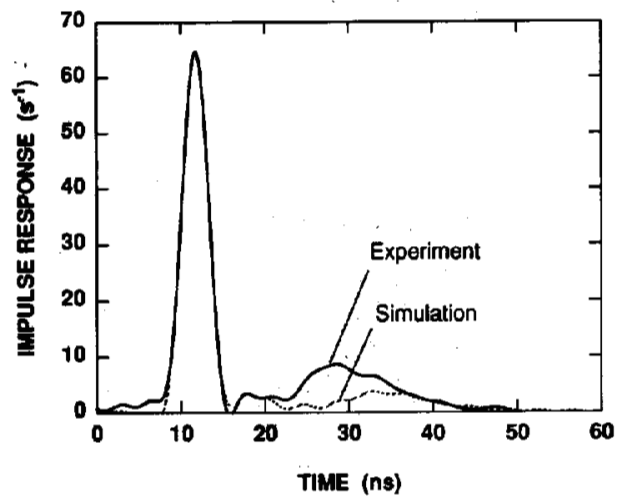

(d)

Fig. 6. Configuration $B$ results. (a) Separate $k$-bounce pulses. (b) Total impulse response. (c) Experimental magnitude response and comparison with simulation. (d) Experimerital impulse response and comparison with simulation.

TABLE II

Summary of Bandwidth and Power Results

\begin{tabular}{|c|c|c|c|c|}
\hline & $\begin{array}{c}\text { Configuration A } \\
\text { (LOS) }\end{array}$ & $\begin{array}{c}\text { Configuration B } \\
\text { (LOS) }\end{array}$ & $\begin{array}{c}\text { Configuration C } \\
\text { (LOS) }\end{array}$ & $\begin{array}{c}\text { Configuration D } \\
\text { (Diffuse) }\end{array}$ \\
\hline $\begin{array}{c}-3 \mathrm{~dB} \text { Bandwidth } \\
\text { (Simulation) }\end{array}$ & $9 \mathrm{MHz}$ & $19.5 \mathrm{MHz}$ & $13 \mathrm{MHz}$ & $32 \mathrm{MHz}$ \\
\hline $\begin{array}{c}-3 \mathrm{~dB} \text { Bandwidth } \\
\text { (Experiment) }\end{array}$ & - & $14 \mathrm{MHz}$ & $12 \mathrm{MHz}$ & $34 \mathrm{MHz}$ \\
\hline $\begin{array}{c}\text { Total Received Power } \\
\text { (Simulation) }\end{array}$ & $2.4 \mu \mathrm{W}$ & $0.31 \mu \mathrm{W}$ & $0.28 \mu \mathrm{W}$ & $0.69 \mu \mathrm{W}$ \\
\hline
\end{tabular}

entire ceiling becomes an effective distributed source, making it difficult for an inadvertent obstruction to cast a sharp shadow onto the receiver.

In Fig. 8(a), we show each of the $k$-bounce impulse responses, $k \in\{1,2,3\}$, as predicted by simulation for configuration $\mathrm{D}$. There is no LOS contribution, so the $k=0$ pulse is identically zero. The combined impulse response is shown in Fig. 8(b); it corresponds to a total received power of $689.8 \mathrm{nW}$ for a $1 \mathrm{~W}$ source. Comparing the received powers for configurations B, C, and D (see Table II), we see that the diffuse system actually provides more power than the LOS systems, despite the lack of a LOS between transmitter and receiver. In fact, this comparison is not completely fair because the nearly Lambertian transmitter radiation pattern is suboptimal as a transmitter for a LOS system, but it is close to optimal as a transmitter for a diffuse system [16]. Nevertheless, the relatively high power provided by the diffuse system combined with it robustness to shadowing makes it an attractive candidate for system design.

The experimental and simulation frequency responses for 


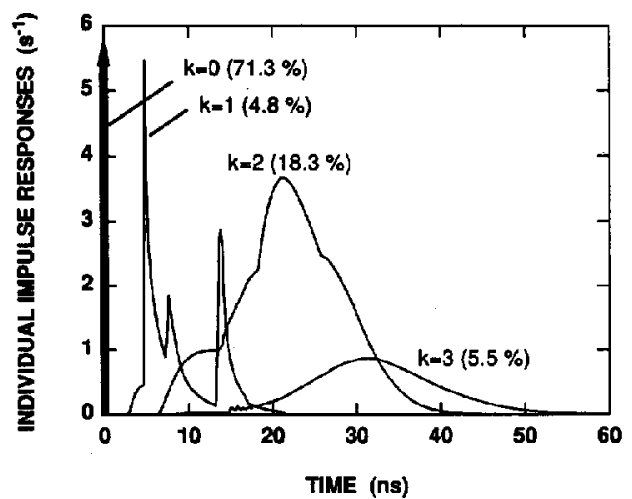

(a)

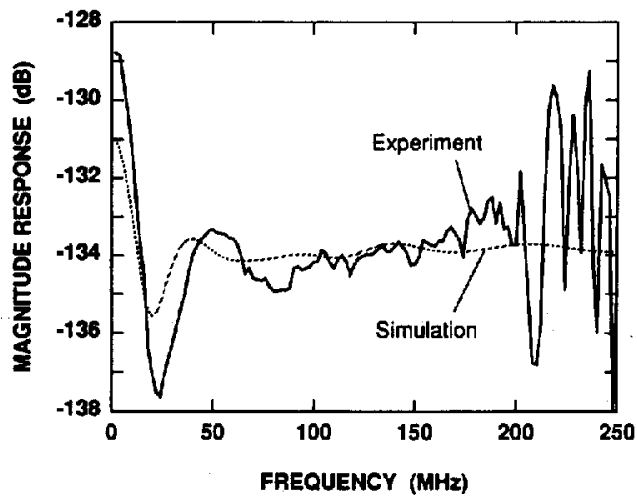

(c)

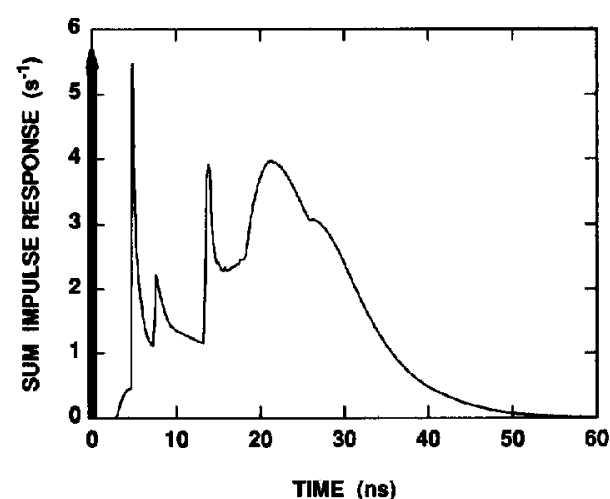

(b)

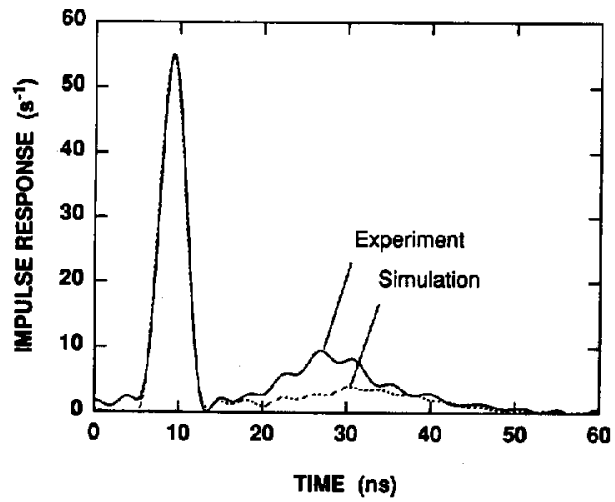

(d)

Fig. 7. Configuration $\mathbf{C}$ results. (a) Separate $k$-bounce pulses. (b) Total impulse response. (c) Experimental magnitude response and comparison with simulation. (d) Experimental impulse response and comparison with simulation.

configuration D are compared in Fig. 8(c). Because there is no LOS contribution, the LOS-based calibration procedure discussed in the previous section cannot be used directly. Instead, we equated the maximum values of the time-domain signals, which in this case are due to first-order reflections. This has been arbitrarily done for all curves to facilitate comparison of simulation and experiment. This procedure, although justified for the LOS configurations $\mathrm{B}$ and $\mathrm{C}$, is less so for configuration $\mathrm{D}$ because it is based on the assumption that the first-order reflector, the ceiling, is ideal Lambertian.

The agreement between simulation and experiment in Fig. 8 is good, probably because of the dominant role played by the first-order reflection as shown in Fig. 8(a). We found the higher-order reflections play little role in determining the shape of the channel frequency response, so that the simulation curve of Fig. 8(c) does not change appreciably when only one bounce is considered.

The experimental $-3 \mathrm{~dB}$ bandwidth of $34 \mathrm{MHz}$ is in good agreement with the simulation bandwidth of $31.8 \mathrm{MHz}$. As compared in Table II, this bandwidth is over twice the bandwidth of each of the LOS systems. The $-3 \mathrm{~dB}$ bandwidth is not a fair metric for comparison, however, for the frequency responses of the LOS systems [see Fig. 4(a), Fig. 6(c), and Fig. 7(c)] exhibit a narrow peak near dc but never roll. off significantly even at high frequencies. This characteristic is common to all impulse responses of the form

$$
h(t)=h_{0} \delta(t)+p(t)
$$

with frequency response $H(\omega)=h_{0}+P(\omega)$, where $P(\omega)$ is a lowpass response. As frequency approaches infinity, $P(\omega)$ becomes negligible, and the frequency response approaches an asymptote of $h_{0}$. The diffuse system, on the other hand, has no LOS Dirac impulse, and so its frequency response rolls off steadily at high frequencies.

The experimental time-domain impulse response is compared to simulation in Fig. 8(d), using the same procedure as that for Fig. 6(d). There, we see that the pulse is wider than the 4 ns resolution of the $250 \mathrm{MHz}$ window, indicating a broad underlying pulse.

\section{Multipath-INDUCED POWER PENALTy}

To illustrate the adverse effects of multipath dispersion on system performance, consider the model for a baseband on-off keyed (OOK) system shown in Fig. 9. The symbols $a_{k} \in\{0,1\}$ are passed through a transmit filter with impulse response $A b(t)$ at a bit rate of $1 / T$, where $A$ is proportional to the average optical intensity of the transmitter. The output 


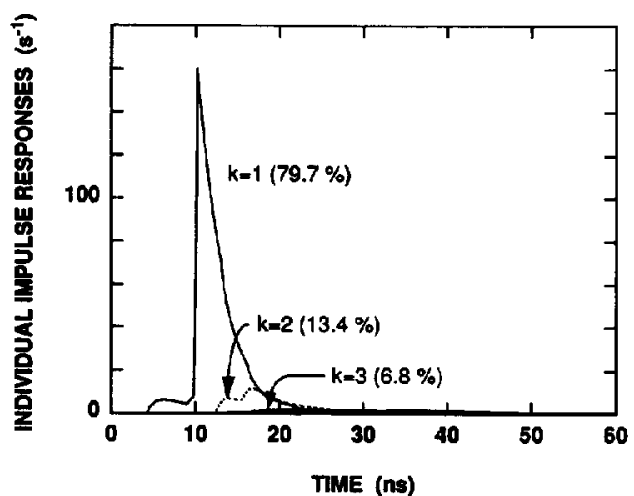

(a)

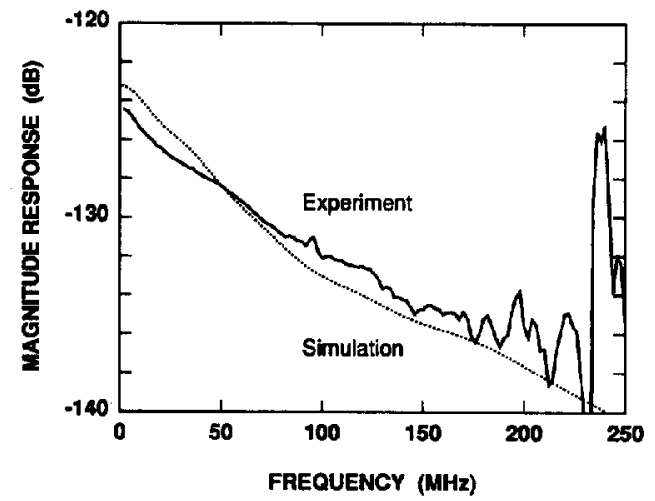

(c)

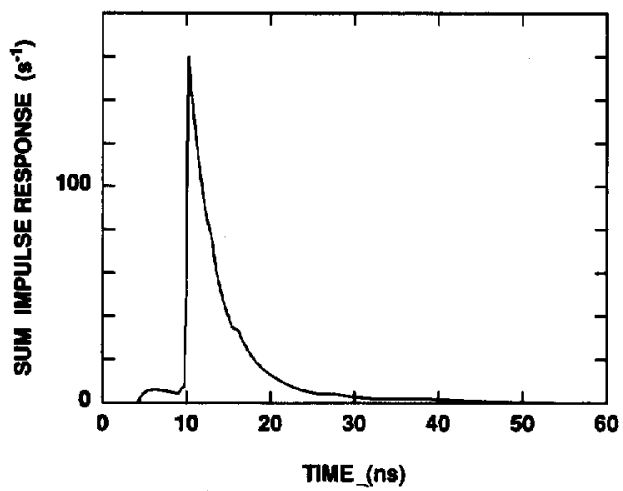

(b)

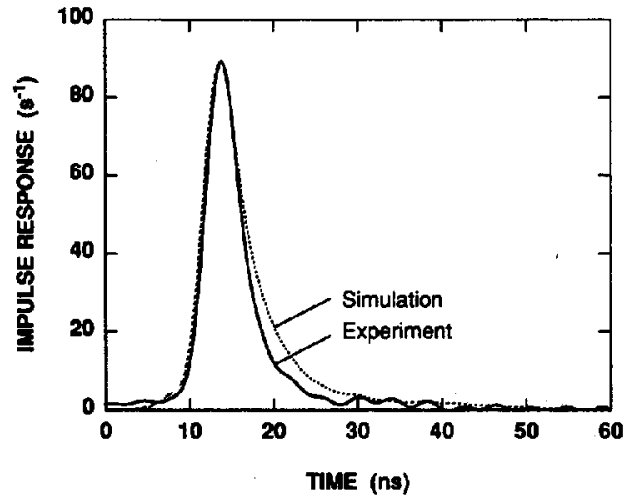

(d)

Fig. 8. Configuration D results. (a) Separate $k$-bounce pulses. (b) Total impulse response. (c) Experimental magnitude response and comparison with simulation. (d) Experimental impulse response and comparison with simulation.

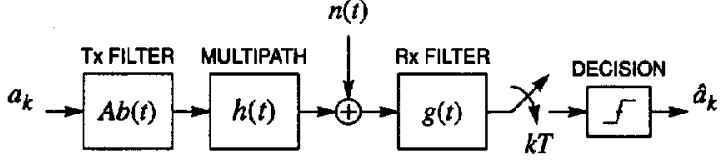

Fig. 9. Baseband OOK system.

of the transmit filter, which represents the intensity of the transmitted signal, is passed through the multipath channel with impulse response $h(t)$. We assume, in this section, that $h(t)$ is normalized to have unity area so that $H(0) \approx 1$. With this assumption, $A$ becomes the average received optical power. The additive noise $n(t)$ represents the shot noise due to ambient light and is accurately modeled as a Gaussian random process [3]. The received signal plus noise is passed through a receive filter with impulse response $g(t)$, sampled at the bit rate, and quantized to yield the estimate $\hat{a}_{k}$ of the $k$ th transmitted bit. We assume that both $b(t)$ and $g(t)$ are normalized to have area $T$, and that $g(t)$ is a Nyquist pulse [19].

The bit error rate for this system can be calculated as follows. First, we note that $y_{k}$, the input to the decision device, can be expressed as

$$
y_{k}=A a_{k} \otimes h_{k}+n_{k}
$$

where the symbol $\otimes$ denotes convolution, $h_{k}$ is the equivalent discrete-time impulse response of the system given by

$$
h_{k}=\left.b(t) \otimes h(t) \otimes g(t)\right|_{t=k T}
$$

and $n_{k}$ is given by

$$
n_{k}=\left.n(t) \otimes g(t)\right|_{t=k T} .
$$

To isolate the power penalty due to intersymbol interference (ISI), we make two assumptions. First, we assume perfect timing recovery; in other words, we assume that the time origin is shifted so as to maximize $h_{0}$. Second, we assume an optimal decision threshold. Basic symmetry arguments lead to the conclusion that this optimal threshold is $A / 2$.

We can rewrite $h_{k}$ as $h_{k}=h_{0} \delta_{k}+\left(1-\delta_{k}\right) h_{k}$, where the second term represents the impulse response "tail," and $\delta_{k}$ is a unit impulse. In general, there will be both precursor and postcursor ISI so the impulse response tail is not necessarily causal.

For a given sequence of bits $a_{k} \equiv\left(\cdots a_{k-1}, a_{k}, a_{k+1} \cdots\right)$, the probability that the $k$ th bit estimate $\hat{a}_{k}$ is in error is given by

$$
\operatorname{Pr}\left[\text { error } \mid a_{k}\right]=Q\left(\rho\left(1-X_{k}\right)\right)
$$


where $Q$ is the Gaussian $Q$-function [19], $\rho \equiv A /(2 \sigma), \sigma^{2}$ is the variance of $n_{k}$, and $X_{k}$ represents the ISI

$$
X_{k}=2 \sum_{i \neq k} a_{i} h_{k-i}
$$

The total bit error rate can be found by averaging over all possible bit sequences:

$$
\begin{aligned}
\mathrm{BER} & =E\left[Q\left(\rho\left(1-X_{k}\right)\right)\right] \\
& =\frac{1}{2^{M}} \sum_{\boldsymbol{a}} Q\left(\rho\left(1-X_{k}\right)\right)
\end{aligned}
$$

where the expectation is taken over $a_{k}$, the elements of which we assume are independent and uniform on $\{0,1\}$. Here, $M$ is the length of the impulse response tail $\left(1-\delta_{k}\right) h_{k}$, assuming it is finite, and the summation is performed over all $\boldsymbol{a} \in\{0,1\}^{M}$.

If there was no multipath dispersion, then $X_{k}$ would be identically zero and BER would reduce to $Q(\rho)$. The value of $\rho$ required to achieve a desired BER of $B E R_{0}$ would then be $\rho_{0}=Q^{-1}\left(B E R_{0}\right)$. With dispersion, however, a larger value of $\rho$ is required to achieve $B E R_{0}$. We, thus, define an optical power penalty as the increased optical signal power required to overcome the multipath ISI and achieve a given $B E R_{0}$ :

$$
\text { power penalty }=10 \log _{10}\left(\frac{\rho \text { required for } B E R_{0}}{Q^{-1}\left(B E R_{0}\right)}\right) \mathrm{dB} \text {. }
$$

We emphasize that this is an optical power penalty; the electrical power penalty, in $\mathrm{dB}$, will be larger by a factor of two.

In Fig. 10, we plot the optical power penalty versus bit rate $1 / T$ for each of the four configurations of Table I using the $K=3$ simulated impulse responses of Fig. 3, Fig. 6(b), Fig. 7(b), and Fig. 8(b). We assume a simple OOK system with an integrate-and-dump receiver, so that the transmitter and receiver filters are identical rectangular pulses $b(t)=g(t)=$ $\operatorname{rect}(2 t / T-1)$, where rect $(t)$ is defined by (9). This receive filter, being matched to the transmit filter, is optimal only when there is no multipath. The results of Fig. 10, thus, illustrate the performance when multipath is ignored in the system design; equalization can improve performance.

We see that, in all cases, the power penalties are significant for bit rates above $10 \mathrm{Mb} / \mathrm{s}$. Configurations $\mathrm{A}, \mathrm{B}$, and $\mathrm{C}$, which are all LOS systems, are seen to be less susceptible to multipath interference. Configuration $\mathrm{A}$ has a higher penalty than $B$ or $\mathbf{C}$ for two reasons: 1) the walls in configuration $A$ are highly reflective; and 2) the receiver is on the floor, so a large fraction of the area of the walls are within its field of view. The power penalty for the diffuse system, labeled $\mathrm{D}$ in the figure, eventually grows much faster with increasing bit rate than any of the LOS systems. Interestingly, however, at moderate bit rates (below $100 \mathrm{Mb} / \mathrm{s}$ ) the power penalty's rate of growth is less than the LOS systems. This is due to the relatively low signal energy carried by second- and third-order reflections for configuration $\mathrm{D}$, as compared to the LOS systems.

For bit rates above $100 \mathrm{Mb} / \mathrm{s}$, the power penalties for two of the LOS systems, $\mathrm{B}$ and $\mathrm{C}$, are seen to decrease as the bit rate

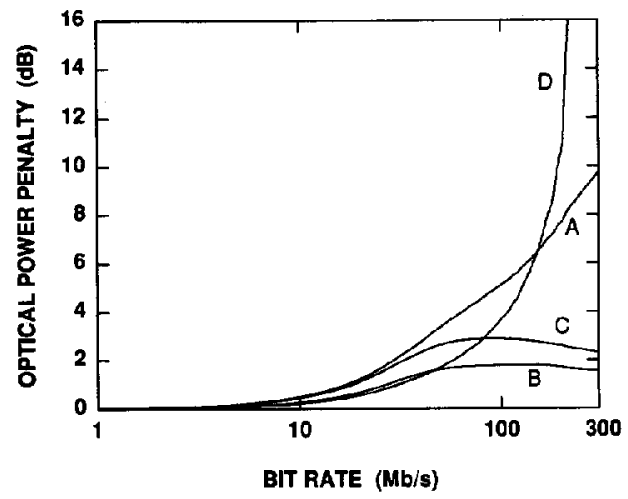

Fig. 10. Optical power penalty versus bit rate for configurations $A$ through D, accounting for up to $K=3$ bounces $\left(B E R_{0}=10^{-6}\right)$.

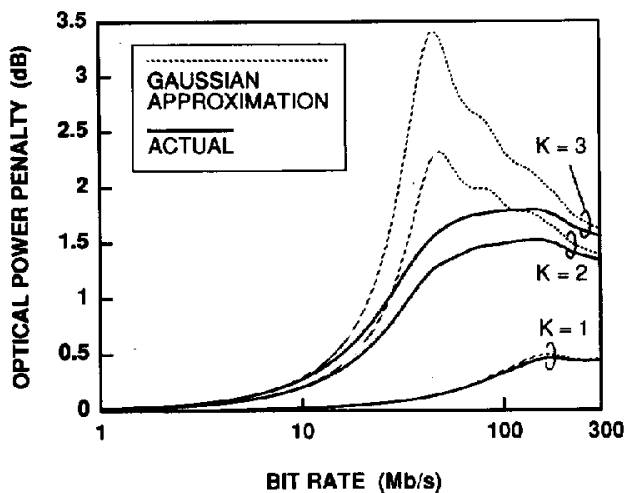

Fig. 11. Comparison between Gaussian approximation and actual power penalty for configuration $\mathrm{B}\left(B E R_{0}=10^{-6}\right)$

increases. This phenomenon is due to the LOS Dirac delta function in the impulse response, and hence does not occur for diffuse systems. We now introduce a second procedure for calculating the optical power penalty based upon a Gaussian approximation, which is useful in explaining the power penalty behavior at high bit rates.

The ISI term $X_{k}$ as defined in (21) is a random variable with mean

$$
\mu=E\left[2 \sum_{i \neq k} a_{i} h_{k-i}\right]=\sum_{i \neq 0} h_{i}=1-h_{0}
$$

and variance

$$
E=\operatorname{var}\left[2 \sum_{i \neq k} a_{i} h_{k-i}\right]=\sum_{i \neq 0} h_{i}^{2} .
$$

Note that $E$ is just the energy contained in the impulse response tail. In (25), we make use of the assumption that $\Sigma h_{k}=1$.

As the bit rate $1 / T$ approaches infinity, the length of the discrete-time impulse response $h_{k}$ also approaches infinity. Therefore, because $\left\{a_{i}\right\}$ are independent, the central limit 
theorem tells us that $X_{k}$ tends towards a Gaussian random variable with mean $\mu$ and variance $E$. With this Gaussian assumption, and rewriting the expectation of (22) as an integral with a Gaussian density function, we find that the BER reduces to

$$
B E R=Q\left(\frac{\rho h_{0}}{\sqrt{1+\rho^{2} E}}\right)
$$

Equating the argument of the $Q$-function here with $\rho_{0}$ and solving for $\rho / \rho_{0}$, the optical power penalty of (24) reduces to the following under the Gaussian assumption

$$
\text { power penalty }=-5 \log _{10}\left(h_{0}^{2}-\rho_{0}^{2} E\right) \mathrm{dB}
$$

where, again, $\rho_{0}=Q^{-1}\left(B E R_{0}\right)$.

In Fig. 11, we compare this approximation with the true power penalty for configuration $B$. The curves are labeled by the maximum number $K$ of reflections considered. The approximate curves, shown with dashed lines, exhibit a more pronounced maximum near $40 \mathrm{Mb} / \mathrm{s}$ than do the actual curves. Since configuration B is a LOS system, $h_{0}$ will approach asymptotically a nonzero constant as $1 / T$ approaches infinity. The energy in the tail, on the other hand, is asymptotically zero when 1/T approaches both zero and infinity, and achieves a maximum somewhere in between. The frequency at which the tail energy is maximum is close to the frequency at which the power penalty achieves its maximum. (The maxima may not coincide exactly when the higher-order reflections arrive soon after the LOS impulse).

The Gaussian approximation is seen to be inaccurate at moderate frequencies near $40 \mathrm{Mb} / \mathrm{s}$, the reason being that, at this bit rate, the length of the impulse response tail is not sufficient for application of the central limit theorem. As the bit rate increases above $100 \mathrm{Mb} / \mathrm{s}$, however, the Gaussian approximation converges to the actual power penalty. From Fig. 6(a), we see that $h_{0}=0.779$ for the $K=3$ impulse response, and so from (28) with $E=0$ we calculate the highbit-rate asymptote of the $K=3$ curve in Fig. 11 to be 1.1 $\mathrm{dB}$.

All three cases $K \in\{1,2,3\}$ are shown in Fig. 11 to illustrate the importance of the higher-order reflections on system performance. The curve labeled $K=1$ accounts for only first-order reflections and is seen to grossly underestimate the true power penalty. The $K=2$ curve is more accurate, but still underestimates the power penalty by as much as $0.4 \mathrm{~dB}$. The curve labeled $K=3$ is identical to the curve labeled $B$ in Fig. 10. Reflections of order greater than 3 will further increase the power penalty, although to a lesser extent. We thus conclude that, since most of the power penalty is due to reflections of order greater than one, the high-order reflections are the dominant source of intersymbol interference for configuration $B$.

\section{SUMMARY}

We have presented a method for evaluating the impulse response of an arbitrary room with Lambertian reflectors. This method can account for any number of reflected paths. A simple algorithm suitable for computer implementation has been presented. The results of computer simulations indicate that reflections of multiple order are a significant source of intersymbol interference for an indoor optical communication system. Our simulations were verified by experimental measurements. The design of a high-speed indoor communication link using infrared will require careful attention to the multipath response described in this paper.

Our experimental results are applicable only to the particular room configurations specified in Table $\mathbf{I}$, so we cannot make general statements about all room configurations. Future work in channel characterization should concentrate on filling this gap, in particular by examining the effects of irregularly shaped rooms, furniture, non-Lambertian and specular reflectors, and shadowing. From a systems design standpoint, a statistical characterization of the channel under various conditions would be useful.

\section{REFERENCES}

[1] F. R. Gfeller, H. R. Müller, and P. Vettiger, "Infrared communication for in-house applications," in Proc. IEEE COMPCON, Washington, D.C., Sept. 5-8, 1978, pp. 132-138.

[2] F. R. Gfeller and U. H. Bapst, "Wireless in-house data communication via diffuse infrared radiation," Proc. IEEE, vol. 67, no. 11, pp. 1474-1486, Nov. 1979.

[3] J. R. Barry, J. M. Kahn, E. A. Lee, and D. G. Messerschmitt, "Highspeed nondirective optical communication for wireless networks," IEEE Netw. Mag., pp. 44-54, Nov. 1991.

[4] T. Minami, K. Yano, and T. Touge, "Optical wireless modem for office communication," in Proc. Nation. Comput. Conf., 1983, pp. 721-728, 1983

[5] Y. Nakata, J. Kashio, T. Kojima, and T. Noguchi, "In-house wireless communication system using infrared radiation," in Proc. Int. Conf. Comput Commun., 1984, pp. 333-337.

[6] I. A. Parkin and J. Zic, "An application of infra-red communications," $J$. Electr. Electron. Eng., Australia-IE Aust. \& IREE Aust., vol. 4, no. 4, pp. 331-336, Dec. 1984

[7] OP. Takahashi and T. Touge, "Optical wireless network for office communication," Japan Electron. Rev. Electron. Comput. Telecomm.,

[8] Z. K. Pahlavan, "Wireless intraoffice networks," ACM Trans. Office Inform. Syst., vol. 6, no. 3, pp. 277-302, July 1988.

[9] C. S. Yen and R. D. Crawford, "The use of directed optical beams in wireless computer communications," IEEE GLOBECOM'85, Dec. 2-5. 1985, pp. 1181-1184.

[10] M. D. Kotzin and A. P. van den Heuvel, "A duplex infra-red systems for in-building communications," IEEE VTC ' 86 , pp. 179-185.

[11] Y. Yamauchi, M. Sato, and T. Namekawa, "In-house wireless optical digital SSMA," Electron. and Commun. in Japan, part 1, vol. 70, no. 6, pp. 87-101, 1987.

[12] T. S. Chu and M. J. Gans, "High speed infrared local wireless communication," IEEE Commun. Mag., vol. 25, no. 8, pp. 4-10, Aug.

[13] D. Hash, J. Hillery, and J. White, "IR roomnet: Model and measurement," IBM Commun. ITL Conf., June 1986.

[14] P. Hortensius, "Research and development plan of the infrared portable data link," internal report, IBM T. J. Watson Res. Cent., Yorktown

[15] A. S. Glassner, Ed., An Introduction to Ray Tracing. San Diego, CA: Academic, 1989.

[16] J. R. Barry, "Wireless communication using non-directed infrared radiation," Ph.D. Diss., Uniy. Calif., Berkeley, 1992.

[17] R. G. Gallager, Information Theory and Reliable Communication. New York: Wiley, 1968.

[18] W. J. Krause, "Experimental characterization of non-directed indoor infrared channels," Master's Rep., Univ. Cal., Berkeley, 1993.

[19] E. A. Lee and D. G. Messerschmitt, Digital Communication. Boston, MA: Kluwer Academic, 1988. 


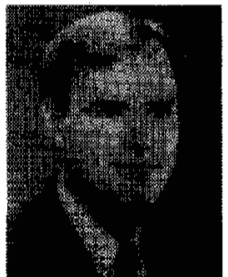

John R. Barry received the B.S. degree (summa cum laude and departmental honors) from the State University of New York at Buffalo in 1986, the M.S. and Ph.D. degrees from the University of California at Berkeley in 1987 and 1992, all in electrical engineering.

He received the 1992 David J. Griep Memorial Prize and the 1993 Eli Jury Award from U.C. Berkeley. Since 1992, he has been an Assistant Professor with the School of Electrical Engineering at the Georgia Institute of Technology, where his research interests include digital communication theory, fiber-optic communications, and broadband wireless communications.

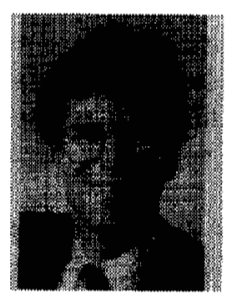

Joseph M. Kahn received the A.B.; M.A., and Ph.D. degrees in physics from the University of California, Berkeley, in 1981, 1983, and 1986, respectively.

He is Associate Professor in the Department of Electrical Engineering and Computer Sciences at UC Berkeley. From 1987 to 1990, he was a Member of Technical Staff in the Lightwave Communications Research Department of AT\&T Bell Laboratories, Holmdel, NJ, where he performed research on multigigabit/second coherent optical fiber transmission systems and related device and subsystem technologies. $\mathrm{He}$ demonstrated the first BPSK-homodyne optical fiber transmission system, and achieved world records for receiver sensitivity in multigigabit/second systems. He joined the faculty of UC Berkeley in 1990, where his research interests include optical fiber communication networks and transmission systems, indoor local area networks using free-space optical communication, and optical interconnects in digital systems. He is a recipient of the National Science Foundation Presidential Young Investigator Award.

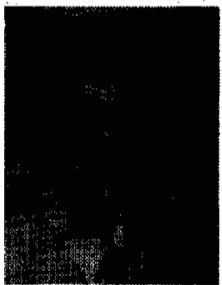

William J. Krause was born in New York City in 1968 . He received the B.S. degree in electrical enginecring from The Johns Hopkins University, Baltimore, MD, in 1990.

He is pursuing the M.S degree in electrical engineering at the University of California, Berkeley. His research areas are communications channels and communications systems.

Mr. Krause is a member of the IEEE Communications Society, Eta Kappa Nu, and Tau Beta $\mathrm{Pi}$
Edward A. Lee received the B.S. degree from Yale University, New Haven, CT, in 1979, the S.M. degree from the Massachusetts Institute of Technology, Cambridge, in 1981, and the Ph.D. degree from the University of California, Berkeley, in 1986

From 1979 to 1982, he was a Member of Technical Staff at Bell Telephone Laboratories, Holmdel, NJ, where he did extensive work with early programmable DSP's and exploratory work in voiceband data modem techniques and simultaneous voice and data transmission. He is currently an Associate Professor in the Department of Electrical Engineering and Computer Sciences, U.C. Berkeley. His research activities include parallel computation, archilecture and software techniques for programmable DSP's, design environments for development of real-time software and hardware, and digital communication. He was a recipient of the 1987 NSF Presidential Young Investigator award, an IBM Faculty Development award, the 1986 Sakrison prize at U.C. Berkeley for the best thessis in Electrical Engineering, and a paper award from the IEEE Signal Processing Socjery. He is coauthor of Digital Communication (Kluwer Academic, 1988) and Digital Signal Processing Experiments (Prentice-Hall, 1989) as well as numerous technical papers. He is Chairman of the VLSI Technical Committee of the Signal Processing Society, co-Program chair of the 1992 Application Specific Array Processor Conference, and is on the Editorial Boand of the Journal on VLSI Signal Processing.

David G. Messerschmitt received the B.S. degree from the University of Colorado in 1967, and the M.S. and Ph.D. degrees from the University of Michigan in 1968 and 1971 , respectively.

$\mathrm{He}$ is currently a Professor and Vice Chair for Computer Resources in the Department of Electrical Engineering and Computer Sciences, U.C. Berkeley. Prior to 1977, he was at Bell Laboratories in Holmdel, NJ. His current research interests include signal processing and transport in broadband networks, advanced video services, and computer-aided design of communications and signal processing systems using object-oriented programming methodologies. He has served as an Editor for Transmission of the IEEE TRANSACTIONS ON COMMUNICATIONS, and as a member of the Communications Society's Board of Governors. He has also organized and participated in a number of short courses and seminars devoted to continuing engineering education. He is a Member of the National Academy of Engineering of the United States. 